\title{
Transit through the gut measured by analysis of a single stool
}

\author{
J. H. CUMMINGS ${ }^{1}$ AND H. S. WIGGINS \\ From the Medical Research Council Gastroenterology Unit, Central Middlesex Hospital, London
}

SUMMARY A method is described for measuring transit time through the gut which requires the collection of only one stool. A dose of 20 radio-opaque markers is given to the subject on each of three consecutive days with breakfast, each dose of marker being of a different type. The first stool passed after rising on the fourth day is collected and its marker content analysed. The method has been validated in 15 subjects by comparing it on 36 occasions with mean transit time measured by a continuous marker technique (MTT-C). In 35 of these studies transit measured from a single stool (SST) and MTT-C were significantly correlated $\mathrm{R}=0.78 \mathrm{P}<0.001$. In one study the designated stool contained none of the SST markers. An alternative way of validating the SST method is described by comparing it with the average of the three mean transit times from three separate doses of marker (MTT-S). The average MTT-S agreed very closely with MTT-C in 36 studies $R=0.94 \mathrm{P}<0.001$ and proved a satisfactory alternative method for validating transit techniques. In a total of 66 studies in 22 subjects average MTT-S correlated significantly with SST, $R=0.85 \mathrm{P}<0.001$.

Transit time-that is, the time it takes for a marker to pass through the gut-has been measured using a variety of techniques since the original observations by Hertz using bismuth (Hertz et al., 1907). A number of authors have described methods in which a single dose of marker is given, such as coloured glass beads (Alvarez and Freedlander, 1924), carmine (Mulinos, 1935), seeds, cotton, ball-bearings (Burnett, 1923; Hoelzel, 1930), radio-isotopes (Hansky and Connell, 1962), radio-opaque polyethylene pellets (Hinton et al., 1969), a radiotelemetering capsule (Connell and Rowlands, 1960; Holdstock and Misiewicz, 1970) or a radio-isotope capsule (Rosswick et al., 1967; Kirwan and Smith, 1974) and transit calculated from the appearance of the marker on $x$-ray or in successive stools. These methods require either the collection of faeces for five to seven days or repeated $x$-rays of the subject and at best give a valid measurement of only the mean value for a group (Cummings, et al., 1975).

An accurate estimate of the average time a marker spends in the gut may be obtained by feeding the marker continuously to subjects for several weeks and measuring its output in the stools (Davignon

${ }^{1}$ Present address: Dunn Nutrition Laboratory, Milton Road, Cambridge.

Received for publication 14 November 1975 et al., 1968; Cummings et al., 1975). However, this technique is too exacting for both subject and investigator for routine use. Neither this nor other techniques combine accuracy with convenience for the subject, and those using radio-isotopes or $x$-rays are unsuitable for epidemiological studies.

While looking for a suitable method for use on an epidemiological scale, it was suggested to us that, if separately identifiable markers were given to a subject on successive days, it might be possible to estimate mean transit time by analysis of a single stool. This paper reports the development of this technique for practical use and assesses its accuracy.

\section{Methods}

\section{SUBJECTS}

Twenty-two healthy male subjects aged 21-33 years who were either university students or members of staff took part in the studies. The procedure was fully explained to them and was done with their full consent.

\section{MARKERS}

The four markers used in the study were:

1. Radio-opaque pellets (Portex Ltd.) which were approximately cube shaped, mean weight $31 \mathrm{mg}$, specific gravity (SG) $1 \cdot 25$. 
2. The pellets in (1) were cut into four small pellets of mean weight $7.8 \mathrm{mg}$ and SG $1 \cdot 25$.

3. $1 \cdot 1 \mathrm{~mm}$ sections were cut from radio-opaque tubing, external diameter $3.0 \mathrm{~mm}$ (Portex R4); mean weight $8 \mathrm{mg}$, SG 1.63.

4. $1 \cdot 1 \mathrm{~mm}$ sections were cut from radio-opaque tubing of external diameter $4.5 \mathrm{~mm}$ (Portex R5); mean weight $16 \mathrm{mg}$, SG $1 \cdot 63$.

All four markers could be clearly distinguished in the stool and were interchangeable, as they behaved similarly in the gut (Cummings et al., 1975).

\section{DEFINITION OF TERMS}

Mean transit time (MTT) is the average time marker takes to pass through a system.

$M T T-S$ Where the marker is given as a single dose the MTT is calculated,

$$
\operatorname{MTT}-S(h)=\frac{\sum_{i=1}^{i=n} x_{i} t_{i}}{\sum_{i=1}^{i=n} x_{i}}
$$

where $x_{1}$ is the number of radio-opaque pellets present in a stool passed after time interval $t_{i}$.

$M T T-C$ Where the marker is given continuously to the subject the MTT is calculated from the turnover time, which is the same as the MTT (Zilversmit, 1960).

$$
\text { MTT-C }=\text { Turnover time }=\frac{\begin{array}{c}
\text { Mean marker } \\
\text { pool size }
\end{array}}{\begin{array}{c}
\text { Number of markers } \\
\text { ingested daily }
\end{array}}
$$

Mean marker pool (MMP) is the sum of the products of the number of markers $\left(x_{i}\right)$ and the period of time $\left(t_{i}\right)$ during which that number of markers are present in the gut. The total number of periods is determined by the frequency with which the number of markers present changes either by ingestion or defaecation and includes the final period from the last change to midnight. It is the average number of markers present in the gut during the day in question.

$$
\text { MMP }=\frac{\sum_{i=1}^{i=n} x_{i} t_{i}}{i=\sum_{i=1}^{n} t_{i}}
$$

The denominator should equal 24 .

Single stool transit (SST) is the mean transit time of marker calculated from the analysis of a single (previously nominated) stool.

\section{PROCEDURE}

In order to have an accurate measure of transit with which to compare the SST, mean transit time was estimated in 15 of the subjects by giving each five markers with every meal (15 markers per day) for from four to 12 weeks (MTT-C). Throughout this period all the stools were collected and the number of markers present in each was found by $x$-raying the stool. MTT-C was calculated from the difference between the total marker input and total markers recovered (Cummings et al., 1975). Eight of the subjects were taking an ad lib diet and seven were on normal but controlled diets. While these 15 subjects were taking the continuous marker, they were given on 36 occasions a dose of 20 of a different marker on three successive days with breakfastthat is, three doses each of a different marker. A value for SST was calculated for each stool passed which contained any of these markers.

A further group of 15 subjects (seven of whom had not taken part in the rest of the study) took, on 30 occasions, the three single doses of marker on successive days and collected their stools until all the markers had been recovered. Overall, therefore, the SST method was performed on 66 occasions in 22 subjects. The type of marker given on each day was randomised among the studies.

\section{CALCULATION OF SST}

For each stool which contained markers the average transit time for the markers in the stool was calculated. In order to identify the best way of calculating the data and which stool was the most appropriate to choose, the markers were analysed in two main ways:

1. Two-marker analysis Based on markers given on day 1 and 2 only.

$$
S S T=\frac{t_{1} s_{1}+t_{2} s_{2}}{s_{1}+s_{2}}
$$

$t_{1}=$ time from ingestion of day 1 markers to time stool passed;

$\mathrm{s}_{1}=$ number of markers from day 1 in stool.

$t_{2}=$ time from ingestion of day 2 markers to time stool passed;

$\mathbf{s}_{2}=$ number of markers from day 2 in stool.

Two-marker analysis was done on the first stool passed after rising on day 2,3 , and 4 .

2. Three-marker analysis Based on all three markers given

$$
S S T=\frac{t_{1} s_{1}+t_{2} s_{2}+t_{3} s_{3}}{s_{1}+s_{2}+s_{3}}
$$

$t_{1} s_{1}$ and $t_{2} s_{2}$ as defined above.

$\mathrm{t}_{3}=$ time from ingestion of day 3 markers to time stool passed.

$s_{3}=$ number of markers from day 3 in stool.

Three-marker analysis was done on the first stool passed after rising on day 3,4 , and 5 . 
If the first stool passed after rising on day 3 was, in fact, passed on day 4 or even day 5 , then the results for SST assigned to all three days would be the same.

The studies were also analysed in a second way: if there were only one or two types of marker present in the stool then the calculation was based on all the markers present. If all three types of marker appeared in the stool, then the marker present in the smallest proportion was counted as zero, and the analysis based on the others-that is, SST was calculated by reference to the two markers present in the greatest numbers only. In the rare event of two of the three markers in the stool being present in equal numbers with more of the third marker present, then the calculation was based on all three markers.

\section{Results}

The mean transit time measured by the continuous marker method (MTT-C) has been used as the reference by which to compare values for transit obtained by the simpler SST technique. The average value for MTT-C obtained for this period during which the SST markers were excreted is used for the comparison. During these studies, the SST markers were given on 36 occasions. SST has been calculated from the markers present in each of the designated stools -that is, those passed after rising on day 2, 3, 4, and 5-and the data for each day correlated with the MTT-C values. The Table shows the correlation coefficient and regression equation for each group of SST values compared with the MTT-C. From this, it may be seen that the day 4 stool combines the best correlation and regression data when analysed by the three-marker method; $r=0.78$ $y=0.66 x+18$.
The result for SST is improved by calculating it from the two markers present in the greatest proportion in the stool, rather than all three markers, and this method of analysis has been adopted for the statistical calculations in Figs. 1-3. Figure 1 shows the SST data derived in all 36 studies from the day 4 stool calculated from the two markers present in the greatest numbers compared with the MTT-C. The correlation is highly significant $(\mathrm{P}<0.001)$ and the predicted values for MTT-C from the regression equation are reasonably good at both ends of the range; $24 \mathrm{~h}=34$ and $96 \mathrm{~h}=81$.

In this group of studies the average MTT-C is $65.8 \mathrm{~h} \pm 4.5 \mathrm{SEM}$ (range 35-130) and the average SST (day 4 stool) $60.3 \mathrm{~h} \pm 3.6 \mathrm{SEM}$ (range 31-118).

When the designated stool contained no markers the method was deemed to have 'failed'. The absence of any marker in the stool indicates a transit time of greater than the time from ingestion of the first marker to the time the stool was passed. Therefore, a minimum value for SST can be calculated where a stool contains no marker. Only one stool in the day 4 SST analysis contained no markers. The minimum value SST calculated for this stool was $96 \mathrm{~h}$, at which time the MTT-C was $130 \mathrm{~h}$. This value has been recorded in Figs. 1 and 3 by a dot and arrowed line. It has not been included in the statistical calculations. We have not encountered any normal subject in a wide range of studies who completely excreted a dose of marker between taking it at breakfast one day and before passing their first stool the next day. One of the 'failures' in the day 5 stool analysis was, however, because all the marker had already been passed.

As the continuous marker technique is long and tedious, an alternative method to use in validating

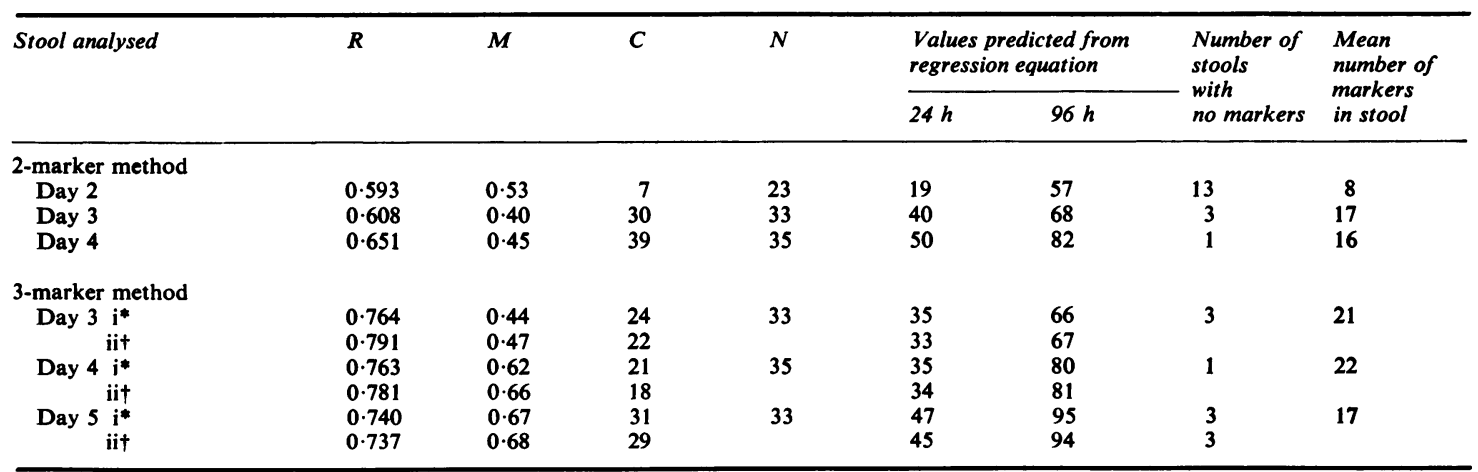

Table Correlation coefficients and regression equations for MTT-C v SST for each designated stool

R: correlation coefficient.

$\mathrm{M}$ : slope of regression line.

$C$ : intercept with $y$ axis.

$\mathbf{N}$ : number of studies included in calculation.

*: values based on analysis of all markers present in stool.

+ values based on analysis of two markers present in largest numbers. 


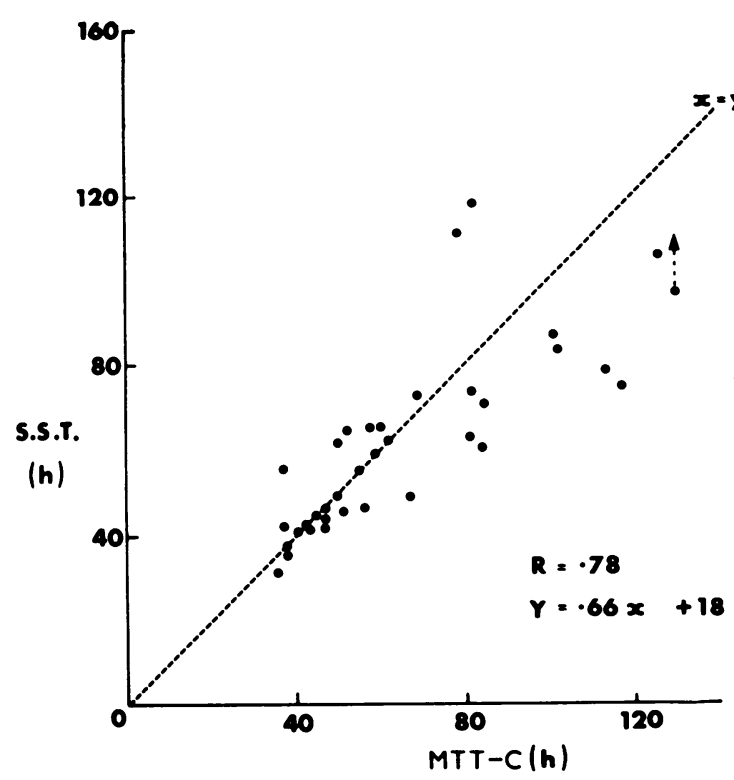

Fig. 1 Average MTT-C during the period in which the SST markers were excreted correlated with the SST from the day 4 stool calculated from the two markers present in the greatest numbers. $N=36$.

transit techniques was looked for. When a single dose of marker is given and is completely recovered in the stools, then the mean transit time may be calculated for that dose (MTT-S). The MTT-S has been

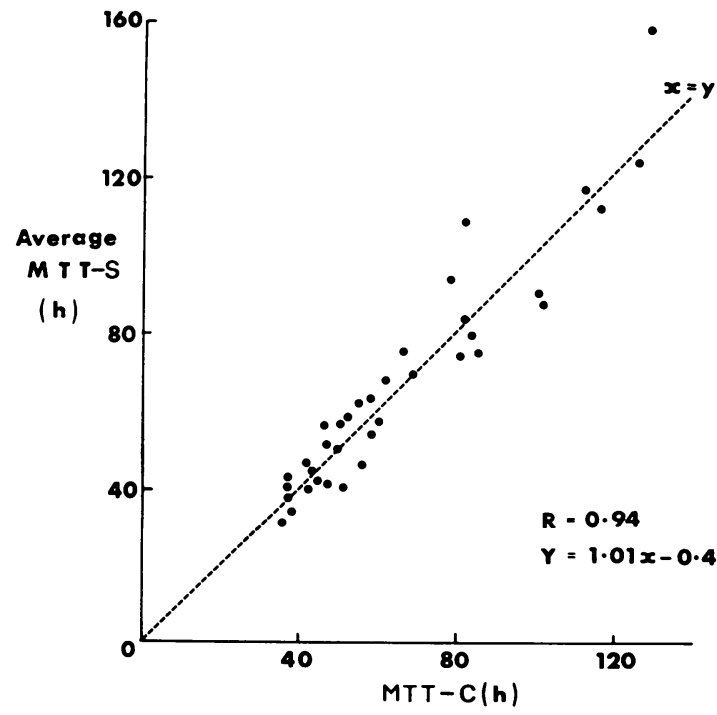

Fig. 2 Average MTT-C (as Fig. 1) correlated with the average of the $3 \mathrm{MTT}-\mathrm{Ss}$ in each study. $N=36$. calculated for each of the three doses of markers in the 36 SST studies and an average of the three MTT-Ss taken. Figure 2 shows the average MTT-S compared with the average MTT-C for the same period in these studies. The agreement between these two methods of measuring transit is excellent and therefore the MTT-S from three consecutive doses provides an alternative and simpler method of validating a transit method.

On this basis, a further 30 SST studies were done in a group of 15 subjects not taking a continuous marker, some of whom had participated in the earlier part of the study. The average MTT-S was calculated and also the SST (day 4 stool) for each subject. In Fig. 3 are shown the average MTT-Ss plotted against the day 4 stool SST for the whole of both groups studied; 66 SSTs in 22 persons. It may be seen that transit time is predicted with reasonable accuracy by the SST method compared with data derived from a seven to 10 day faecal collection. Only one stool in the whole group contained no markers, as already indicated.

\section{Discussion}

On the basis of these results, transit time through the gut may be found by collecting and analysing only one stool by the following procedure:

1. A dose of 20 markers is given to the subject on three successive days with breakfast, using a different but comparable marker on each day.

2. The first stool passed after rising on the fourth

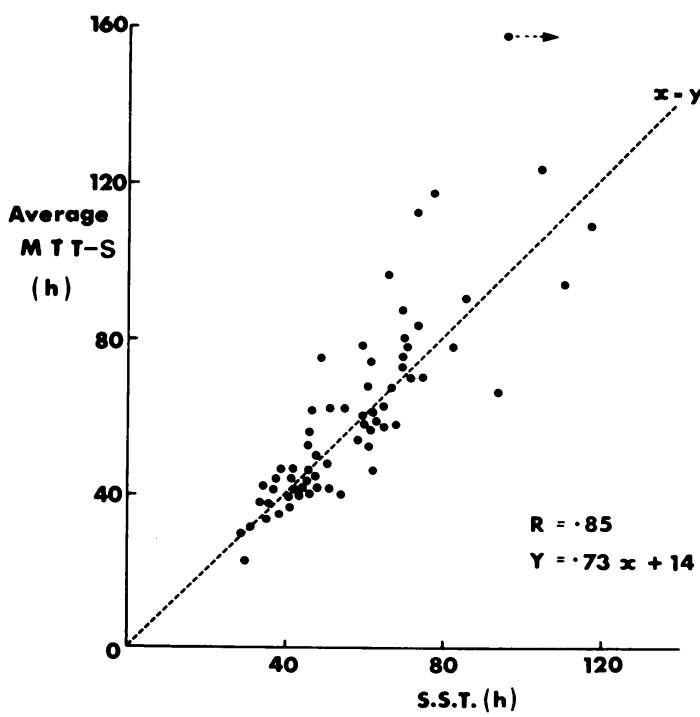

Fig. 3 SST from day 4 stool (as Fig. 1) correlated with the average MTT-S in all studies. $N=66$. 
day is collected and the amount of each marker present is estimated.

3. The data is substituted in the equation

$$
\mathrm{SST}=\frac{\mathrm{t}_{1} s_{1}+t_{2} s_{2}}{s_{1}+s_{2}}
$$

where $t_{1}$ and $t_{2}$ are the time in hours from the ingestion of the two markers present in the greatest number in the stool to the time the stool is passed, and $s_{1}$ and $s_{2}$ the number of each marker present. If no stool is passed on day 4 , then the first stool to be passed either on day 5,6 , or whenever passed is analysed.

While this method has been developed particularly with convenience and simplicity in mind for use in epidemiological studies, it nevertheless gives an accurate estimate of transit in the range studied. Once transit times begin to exceed four days, then its accuracy falls off. However, most published data on transit in normal people fall into this range. In a population where the average transit time is of the order of six days or more, such as constipated subjects (Hinton and Lennard-Jones, 1968; Eastwood, 1972), then selection of a later stool should prove optimal for analysis.

The range of the method may also be extended by increasing the number of days on which markers are given and collecting the first stool passed on the day after the final dose of markers. This, however, will ultimately be limited by the ability to distinguish the markers in the stool. Markers might also be given on alternate days. The good agreement between the average MTT-S and MTT-C (Fig. 2) suggests that any modified form of the SST procedure may be validated by collecting stools until all the markers have been recovered and correlating this with the designated stool, rather than using the much longer continuous marker method.

The finding that the best estimate of transit time is derived from analysis of the two markers present in the greatest numbers even if three markers are present might be expected from the exponential nature of the excretion of markers given as a single dose, which other workers have found (Davignon et al., 1968). The first few markers excreted will underestimate the MTT and the last few overestimate it. Since the markers in the minority in the stool are likely to fall into one of these categories, it is not surprising that excluding them improves the agreement of the SST with the MTT-C derived by a more tedious but accurate method.

The SST method of measuring transit is accurate over the range one to four days and should be useful for both epidemiological studies and as a hospital outpatient procedure.

The authors are grateful to the volunteers who took part in the study without whose cooperation it would not have succeeded. Dr E. N. Rowlands has given helpful advice and criticism and Mrs Penelope Stuart administered the project for which we are indebted to her. The original idea came to us via a working party on colon cancer of the International Association for Cancer Research (Lyon).

\section{References}

Alvarez, W. C., and Freedlander, B. L. (1924). The rate of progress of food residues through the bowel. Journal of the American Medical Association, 83, 576-580.

Burnett, F. L. (1923). The intestinal rate and the form of the faeces. American Journal of Roentgenology, 10, 599-604.

Connell, A. M., and Rowlands, E. N. (1960). Wireless telemetering from the digestive tract. Gut, 1, 266-272.

Cummings, J. H., Jenkins, D. J. A., and Wiggins, H. S. (1976). Measurement of the mean transit time of dietary residue through the human gut. Gut, 17, 216-218.

Davignon, J., Simmonds, W. J., and Ahrens, E. H. (1968). Usefulness of chromic oxide as an internal standard for balance studies in formula-fed patients and for assessment of colonic function. Journal of Clinical Investigation, 47, 127-138.

Eastwood, H. D. H. (1972). Bowel transit studies in the elderly. Radio-opaque markers in the investigation of constipation. Gerontologia Clinica, 14, 154-159.

Hansky, J., and Connell, A. M. (1962). Measurement of gastrointestinal transit using radio-active chromium. Gut, 3, 187-188.

Hertz, A. F., Morton, C. J., Cook, F., Cox, A. N., Gardiner, H., Schlesinger, E. G., and Todd, A. H. (1907). The passage of food along the human alimentary canal. Guy's Hospital Reports, 61, 389-427.

Hinton, J. M., and Lennard-Jones, J. E. (1968). Constipation: definition and classification. Postgraduate Medical Journal, 44, 720-723.

Hinton, J. M., Lennard-Jones, J. E., and Young, A. C. (1969). A new method for studying gut transit times using radioopaque markers. Gut, 10, 842-847.

Hoelzel, F. (1930). The rate of passage of inert materials through the digestive tract. American Journal of Physiology, 92, 466-497.

Holdstock, D. J., and Misiewicz, J. J. (1970). Factors controlling colonic motility: colonic pressures and transit after meals in patients with total gastrectomy, pernicious anaemia or duodenal ulcer. Gut, 11, 100-110.

Kirwan, W. O., and Smith, A. N. (1974). Gastrointestinal transit estimated by an isotope capsule. Scandinavian Journal of Gastroenterology, 9, 763-766.

Mulinos, M. G. (1935). The value of selective drugs in the treatment of constipation. Review of Gastroenterology, 2, 292-301.

Rosswick, R. P., Stedeford, R. D., and Brooke, B. N. (1967). New methods of studying intestinal transit times. Gut, 8, 195-196.

Zilversmit, D. B. (1960). The design and analysis of isotope experiments. American Journal of Medicine, 29, 832-848. 